\title{
BRIEF
}

\section{Implementation of the Individual Teamwork Observation and Feedback Tool (iTOFT) to Evaluate APPE Student Performance}

\author{
Amanda Margolis, PharmD, MS, Shweta Shah, MS, Joseph A. Zorek, PharmD, Mara Kieser, MS, RPh, Beth \\ Martin, PhD, RPh
}

University of Wisconsin-Madison, School of Pharmacy, Madison, Wisconsin

Corresponding Author: Amanda Margolis, University of Wisconsin-Madison, School of Pharmacy, 777 Highland Ave, Madison, WI 53719. Tel: 608-890-0434. Email: Amanda.Margolis@wisc.edu

Submitted February 6, 2021; accepted June 28, 2021; ePublished July 2021

\begin{abstract}
Objective. A direct observation and rating of students' interprofessional teamwork skills was added as a required activity to an advanced pharmacy practice experience (APPE) curriculum using the Individual Teamwork Observation and Feedback Tool (iTOFT). The objective of this evaluation was to determine the appropriateness and feasibility of iTOFT implementation during APPEs.

Methods. In the academic year 2018-2019, preceptors administered iTOFT at least once during required acute care and ambulatory care APPEs. After administration, which involved direct observation of students' behavior on an interprofessional team, preceptors evaluated iTOFT results and offered feedback to students. To determine implementation success of the iTOFT activity, an implementation satisfaction survey was administered to preceptors and students, which included questions on appropriateness and feasibility.

Results. The iTOFT activity was completed 149 times each during required acute care and ambulatory care APPEs. Results demonstrated positive teamwork behaviors; for example, no students received an 'inappropriate' rating on any iTOFT item. The implementation survey response rate was 33\% (34/102) for preceptors and 40\% (50/125) for students. Both groups reported that the iTOFT activity was feasible. Respondents also reported that completing the iTOFT activity did not interfere with workflow and that it was not difficult to find opportunities to complete it. Per preceptor report, mean time to complete the iTOFT activity was 37.7 minutes, but there were concerns identified about fidelity to all iTOFT steps.

Conclusion. Incorporation of the iTOFT activity into required acute and ambulatory care APPEs was feasible. Future directions include determining ways to increase iTOFT fidelity.

Keywords: experiential education, interprofessional education, assessment, pharmacy
\end{abstract}

\section{INTRODUCTION}

Improvements in interprofessional practice can decrease mortality ${ }^{1,2}$, rehospitalization ${ }^{3}$, and health care costs ${ }^{4}$ along with increased patient satisfaction. ${ }^{5}$ Therefore, it is important students learn interprofessional teamwork and communication prior to graduation. This is reflected in the American Council for Pharmacy Education (ACPE) accreditation standard 11 regarding interprofessional education (IPE). ${ }^{6}$ By graduation, pharmacy students need to be able to contribute to an interprofessional team.

To ensure that all graduates are "team ready," a direct assessment of interprofessional teamwork and communication in a real health care setting would be ideal. ${ }^{7}$ Therefore, a purposeful evaluation of students' interprofessional teamwork was added to the University of Wisconsin-Madison School of Pharmacy (UW-Madison SOP) advanced pharmacy practice experience (APPE) curriculum for required acute and ambulatory care APPEs during the 2018-2019 experiential year. The Individual Teamwork Observation and Feedback Tool (iTOFT) was adopted for preceptors to evaluate and offer feedback to APPE students following a direct observation of their performance on an interprofessional team. ${ }^{8,9}$ The iTOFT is a validated tool and was chosen primarily for three reasons: 1) it is designed to assess an individual's performance on a team, not the entire team's performance; 2) it uses a consistent evaluation scale; and 3 ) it is a relatively short 11 -item evaluation. ${ }^{10}$ Other available validated tools measured student perceptions of interprofessional teamwork, evaluated the performance of a team, or were much longer with a varying scale. ${ }^{10}$

As this was a new rotation requirement across a variety of practice settings, and individual sites and preceptors were tasked with incorporating the iTOFT evaluation into the rotation, an assessment of implementation was conducted. Given the wide range of practice settings and health systems where students complete their experiential rotations, it was important to ensure that rotation sites were 1) able to find student opportunities for interprofessional teamwork and 2) that 
preceptors found the activity acceptable. Implementation research determines the success of the implementation process and can offer an experiential education office a framework to evaluate the extent students and preceptors are able to complete required activities in a satisfactory manner. ${ }^{11}$ By measuring implementation success, outcomes of a new activity are measured; and if not as expected, further investigation can determine whether the intervention or activity is ineffective or the implementation was poor. ${ }^{12}$ Common implementation outcomes include adoption, acceptability, feasibility, fidelity,

and sustainability. ${ }^{13,14}$ Results from implementation research can be used to learn how to better introduce and sustain new activities across multiple practice sites. The objective of this evaluation was to determine implementation appropriateness and feasibility of using the iTOFT to evaluate student interprofessional communication and teamwork during APPE rotations.

\section{METHODS}

During the 2018-2019 experiential year, the iTOFT was implemented for preceptors to document interprofessional teamwork behaviors and give formative feedback to APPE students. The iTOFT offers a validated basic and advanced version. ${ }^{8,9}$ Following a pilot of the tool with five purposefully selected practice sites and thorough discussion with preceptors, the 11-item basic version of the tool was selected. Following a request from an APPE coordinator, the "discusses safety issues" item from the advanced tool was added to the assessment and the version of iTOFT administered in this evaluation included 12 items (See Table 1 for specific items). The evaluation for each skill has four assessment options: 1) not applicable, 2) inappropriate, 3) appropriate, and 4) responsive. Appropriate indicates, "The student is engaged with the team in the activity. However, does not take the opportunity to further develop teamwork [behaviors]." Responsive indicates "The student is actively engaged with the team in the activity and demonstrates commitment in learning about treamwork." "A tool to document the iTOFT evaluation was built by the UW-Madison SOP Instructional Information Technology Department and integrated into the already established online clerkship database. In addition to collecting the items on the iTOFT tool, the other members of the health care team, which students interacted with during the observation, and the mode of team interaction (ie, face-to-face, telemedicine, or telephone) were collected. Other health care providers were designated as practitioners if they held an entry-level practice degree or as students if they did not hold an entry-level practice degree.

Observation of student interprofessional communication and teamwork with feedback and online documentation of the iTOFT was required once during both the acute care and ambulatory care required APPE rotations. Preceptors could complete the tool multiple times but documented it once during the rotation. Completion of the activity was required, but the evaluation by the preceptor did not contribute to the students' final letter grade. However, preceptors were encouraged to consider the students' performance on the iTOFT when completing an IPE item on the final student performance evaluation. A 23-minute online presentation explaining the tool and expectations was available for both students and preceptors to review the purpose, expectation, and items on the tool. The iTOFT activity was also introduced at the 2018 Annual Clinical Instructor meeting and described in the General Experiential Manual and course orientations.

To assess the implementation of the iTOFT activity, the implementation outcomes of appropriateness and feasibility were adopted from the Conceptual Model of Implementation Research. ${ }^{11,13}$ Appropriateness was operationalized as satisfaction with the iTOFT activity and feasibility was operationalized as fit or suitability of the iTOFT activity in practice. This was measured as an implementation satisfaction survey to preceptors and students (items assessed in Table 2). The surveys contained seven and six questions for preceptors and students, respectively, to assess the implementation outcomes using a 4-point response ranging from "Do not agree" to "Completely agree." The questions were adopted from an implementation research question bank and previous implementation research. ${ }^{15}$ Students and preceptors were also asked two additional feasibility questions regarding which steps of the iTOFT activity they completed and how many minutes the activity took to complete. Students and preceptors who completed the iTOFT evaluation by the third rotation after the introduction of the activity were invited to complete the implementation satisfaction survey. The survey was administered online using Qualtrics (Qualtrics Labs Inc., Provo, UT).

To triangulate the iTOFT scores and demonstrate improvement in student IPE performance, the Interprofessional Collaborative Competency Attainment Scale (Revised) or ICCAS-R was administered to students on the required acute and ambulatory care APPEs. ICCAS-R is a validated post-retrospective self-evaluation of interprofessional skills. ${ }^{16,17}$ It uses a scale of: $1=$ Poor; $2=$ Fair; $3=$ Good; $4=$ Very good; $5=$ Excellent. The ICCAS-R survey was administered to students during the last week of the APPE online via Qualtrics (Qualtrics Labs Inc., Provo, UT).

All surveys included a single reminder email 3 to 7 days following the initial invitation to complete the survey. At the end of the academic year, a report was generated using all iTOFT evaluation results. Descriptive statistics were used to characterize results. Reliability tests and paired t-tests were performed on ICCAS-R for acute and ambulatory care settings separately. This way, ICCAS-R student self-evaluation of IPE performance prior to rotation was compared to end of rotation. An alpha level of 0.05 was used to indicate statistical significance using Stata version 14.2. This evaluation was 
certified as a quality assurance project by the University of Wisconsin-Madison Education and Social/Behavioral Science Institutional Review Board.

\section{RESULTS}

There were 149 iTOFT evaluations completed in both the acute care and ambulatory care rotations (100\% of the graduating class). On the acute care rotation $125(84 \%)$ of the observations were face-to-face, $13(9 \%)$ were telephone, and 11 were other or unanswered (7\%). On the ambulatory care rotation $90(60 \%)$ of the observations were face-to-face, $39(26 \%)$ were telephone, and 20 were other or unanswered (13\%). A majority of observed interactions involved a prescriber and were with health care providers holding at least an entry-level degree.

Students were rated well on the iTOFT evaluation (Table 1). No students received an inappropriate rating on any iTOFT item. Students were often rated as responsive (range 54-86\% in acute care and $46-79 \%$ in ambulatory care). In general, preceptors did not use the not applicable rating when completing the iTOFT evaluations.

Of the 102 preceptors who submitted an iTOFT evaluation, $34(33 \%)$ completed the implementation satisfaction survey (20 acute care, 10 ambulatory care, 4 community practice). All preceptors were volunteer preceptors, which is the case for the majority of UW-Madison SOP acute and ambulatory care APPEs. Of the 125 students who had completed an iTOFT evaluation at the time of the survey, 50 students (40\%) responded. Of those who completed the survey question, $75 \%$ of preceptors and $52 \%$ of students reported viewing the iTOFT training. Results of the student and preceptor implementation surveys had similar distributions (Table 2). There were mixed results for the appropriateness implementation outcome, but both students and preceptors reported the iTOFT activity was feasible. Respondents reported completing the iTOFT activity did not interfere with workflow and it was not difficult to find an opportunity to complete the activity. Per preceptor report, mean time to complete the iTOFT activity was 37.7 minutes. In the acute care setting the mean was 50 minutes and in the ambulatory care rotation it was 20 minutes.

While not measured a priori, there was lower than expected fidelity to the steps of the iTOFT activity (Table 3 ). While initially intended to be a feasibility measurement, it was noted that some required steps were not completed and thus a concern that the activity was not being completed as intended. ${ }^{13}$ Of particular concern were the areas of direct observation and feedback within the iTOFT activity where $20 \%$ of preceptors and $40 \%$ of students did not report the preceptor completing direct observation and $30 \%$ of preceptors and $40 \%$ of students did not report giving or receiving feedback.

Response rates for the ICCAS-R included 121 students $(81 \%)$ and 130 students $(87 \%)$, in the acute care and ambulatory care settings, respectively. On comparing pre-rotation to end of rotation mean scores for all 20 ICCAS-R items, students in the acute care setting self-described a statistically significant increase in IPE performance from 3.3 $( \pm 0.67)$ at baseline to an end of rotation mean of $4.1( \pm 0.57 ; p<.001)$. Results were similar in the ambulatory care APPE with a baseline mean of $3.2( \pm 0.66)$ and an end of rotation mean of $4.1( \pm 0.62 ; p<.001)$. There was also a statistically significant increase in all ICCAS-R items for both the acute care and ambulatory care rotations when assessed individually. At the end of the acute care APPE, 58\% of students described their overall ability to collaborate interprofessionally as "much better" and 35.5\% described it as "somewhat better". This was lower in the ambulatory care APPE with $48.2 \%$ selecting "much better" and 40.7\% selecting "somewhat better". The baseline ICCAS-R had high internal consistency with a Cronbach's alpha of 0.96 in the acute care APPE and 0.97 for the ambulatory care APPE.

\section{DISCUSSION}

The inclusion of the iTOFT activity in both required acute care and ambulatory care APPEs appears feasible. All students and preceptors on acute care and ambulatory care submitted the iTOFT activity, suggesting the activity is feasible to incorporate and use in clinical settings. ${ }^{8,10}$ Both preceptors and students reported there were opportunities in practice to complete interprofessional interactions and the activity did not interfere with workflow. While the length of time to complete iTOFT activity in the acute care setting may seem high, this often included the time to observe the student on rounds and was acceptable to the preceptors completing the survey. This is consistent with the work of Crowl and colleagues who introduced the advanced version of the iTOFT to pharmacy preceptors. ${ }^{18}$ After receiving training and trialing the iTOFT, they felt it was easy to use, relevant to practice, and took a reasonable amount of time to complete. ${ }^{18}$

It was not surprising to find that the introduction of the iTOFT activity did not change precepting behaviors or improve student learning as the goal of the activity was to document student interprofessional teamwork behaviors. This was inconsistent with the findings by Crowl and colleagues where preceptors reported the introduction of interprofessional assessment tools increased the frequency of feedback they shared which then improved student interprofessional behaviors. ${ }^{18}$ This may be because preceptors in that study used the interprofessional assessments several times over the course of an APPE whereas the UW-Madison SOP only required the iTOFT activity be documented once during the required acute and ambulatory care rotations. While the implementation satisfaction survey suggests some 
preceptors were not satisfied with the additional assessment activity, the iTOFT activity can document student-readiness for practice, thus meeting needs of the pharmacy school. Anecdotally, the preceptors who were less satisfied with the additional assessment tended to be those who had already incorporated IPE activities into their rotation.

An open-ended question was included on the implementation satisfaction survey and may elucidate why some preceptors appreciated the addition of the iTOFT activity. Some preceptors described increased emphasis on the value of interprofessional collaboration and providing structure to their student feedback on interprofessional teamwork. One preceptor wrote "Students were completing interprofessional activities prior to the iTOFT, but now this gives a formal structure for feedback". This was consistent with Crowl and colleagues who also described pharmacy preceptors reporting an increase in the structure of their feedback when they started using validated interprofessional assessment tools. ${ }^{18}$

There are several limitations to this evaluation. This evaluation was observational and only assessed after the iTOFT activity had started. While the implementation satisfaction survey was reviewed for understanding by the investigators, it was not a validated survey. Lastly, while there was a high rate of completion for the iTOFT activity and the ICCAS-R, there was a low preceptor and student response rate to the implementation satisfaction survey portion of the evaluation. This may be due to the lack of requirement or incentive associated with this survey.

The low fidelity to some iTOFT activity steps that both students and preceptors reported as not completed was concerning. Most notably a specific direct observation or discussion of the iTOFT results had a low rate of reported completion. The low rate of training completion may have contributed to lower than anticipated fidelity, especially for students, and the training was made a required part of APPE orientation the subsequent year. Fidelity is also an ongoing future direction to identify why steps are being omitted and how a school can better promote the activity or make it easier to complete.

\section{CONCLUSION}

Incorporation of the iTOFT activity into required acute and ambulatory care APPEs was feasible and achieved the programmatic goal of documenting pre-graduation readiness of students to perform on interprofessional teams. Future directions include determining ways to increase fidelity to the program.

\section{ACKNOWLEDGMENTS}

The authors acknowledge and thank Kate Hartkopf, PharmD for her review of the assessment plan; Mike Pitterle, MS, RPh and Kathy Chylla, BS for building the electronic iTOFT tool; and Robert Breslow, RPh and Denise Pigarelli, PharmD for their support in the implementation of the iTOFT activity.

\section{REFERENCES}

1. Martin JS, Ummenhofer W, Manser T, Spirig R. Interprofessional collaboration among nurses and physicians: making a difference in patient outcome. Swiss Med Wkly. 2010;140:w13062. doi:10.4414/smw.2010.13062

2. Kim M, Barnato AE, Angus DC, Fleisher LA, Kahn JM. The effect of multidisciplinary care teams on intensive care unit mortality. Arch Intern Med. 2010;170(4):369-376. doi:10.1001/archinternmed.2010.124

3. Boykin A, Wright D, Stevens L, Gardner L. Interprofessional care collaboration for patients with heart failure. Am J Heal Pharm. 2018;75(1):e45-e49. doi:10.2146/ajhp160318

4. Madan A, Borckardt JJ, Barth KS, Romagnuolo J, Morgan KA, Adams DB. Interprofessional collaborative care reduces excess service utilization among individuals with chronic pancreatitis. J Healthc Qual. 2013;35(5):41-46. doi:10.1111/jhq.12025

5. Ashcraft S, Bordelon C, Fells S, George V, Thombley K, Shirey MR. Interprofessional clinical rounding: effects on processes and outcomes of care. J Healthc Qual. 2017;39(2):85-94. doi:10.1097/JHQ.0000000000000039

6. Accreditation Council for Pharmacy Education. Guidance for the Accreditation Standards and Key Elements for the Professional Program in Pharmacy Leading to the Doctor of Pharmacy Degree. February 2, 2015. Accessed February 6, 2021. https://www.acpe-accredit.org/pdf/GuidanceforStandards2016FINAL.pdf

7. Fromme H, Karani R, Downing S. Direct Observation in medical education: review of the literature and evidence for validity. Mt Sinai J Med. 2009;76(4):365-371. doi:10.1002/msj.20123.

8. Thistlethwaite J, Dallest K, Moran M, et al. Introducing the individual Teamwork Observation and Feedback Tool (iTOFT): development and description of a new interprofessional teamwork measure. J Interprof Care. 2016;30(4):526-528. doi:10.3109/13561820.2016.1169262

9. Thistlethwaite J, Dallest K, Bainbridge L, et al. Work-based assessment of teamwork: an interprofessional approach. 2015. Accessed February 6, 2021. http://derby.openrepository.com/derby/bitstream/10545/621207/1/ID122193_UQ_Thistlethwaite_Final+report_2015.pdf 
10. Shrader S, Farland MZ, Danielson J, Sicat B, Umland EM. A systematic review of assessment tools measuring interprofessional education outcomes relevant to pharmacy education. Am J Pharm Educ. 2017;81(6):119. doi:10.5688/ajpe816119

11. Proctor EK, Landsverk J, Aarons G, Chambers D, Glisson C, Mittman B. Implementation research in mental health services: an emerging science with conceptual, methodological, and training challenges. Adm Policy Ment Heal Ment Heal Serv Res. 2009;36(1):24-34. doi:10.1007/s10488-008-0197-4

12. Curran GM, Bauer M, Mittman B, Pyne JM, Stetler C. Effectiveness-implementation hybrid designs. 2012;50(3):217-226. doi: 10.1097/MLR.0b013e3182408812

13. Proctor E, Silmere H, Raghavan R, et al. Outcomes for implementation research: conceptual distinctions, measurement challenges, and research agenda. Adm Policy Ment Heal Ment Heal Serv Res. 2011;38(2):65-76. doi:10.1007/s10488-010-0319-7

14. Glasgow RE, McKay HG, Piette JD, Reynolds KD. The RE-AIM framework for evaluating interventions: what can it tell us about approaches to chronic illness management? Patient Educ Couns. 2001;44(2):119-127. doi:10.1016/S0738-3991(00)00186-5

15. CFIR ResearchTeam. Consolidated Framework for Implementation Research interview guide Tool. http://www.cfirguide.org/participate.html

16. Archibald D, Trumpower D, MacDonald CJ. Validation of the interprofessional collaborative competency attainment survey (ICCAS). J Interprof Care. 2014;28(6):553-558. doi:10.3109/13561820.2014.917407

17. Schmitz CC, Radosevich DM, Jardine P, MacDonald CJ, Trumpower D, Archibald D. The Interprofessional Collaborative Competency Attainment Survey (ICCAS): a replication validation study. J Interprof Care. 2017;31(1):28-34. doi:10.1080/13561820.2016.1233096

18. Crowl AN, Burkhardt C, Shrader S. Potential best practices for assessment of interprofessional team-ready behaviors on APPEs. Curr Pharm Teach Learn. 2020;12(2):156-162. doi:10.1016/j.cptl.2019.11.010 
Table 1. iTOFT Evaluations by Preceptor; $n=149$ [n (\%)]

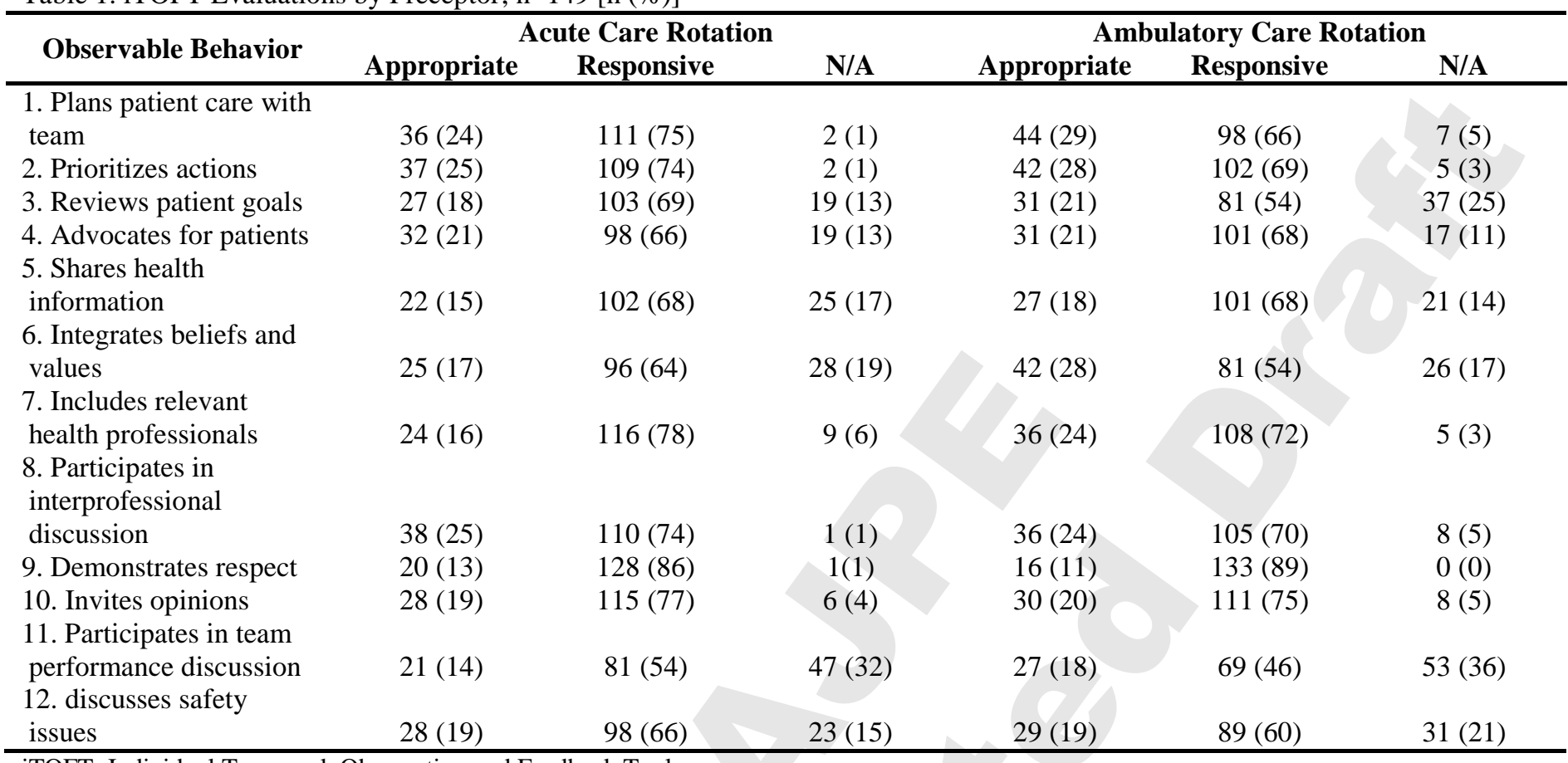

iTOFT=Individual Teamwork Observation and Feedback Tool 
Table 2. Implementation Satisfaction Survey Results [n (\%)]

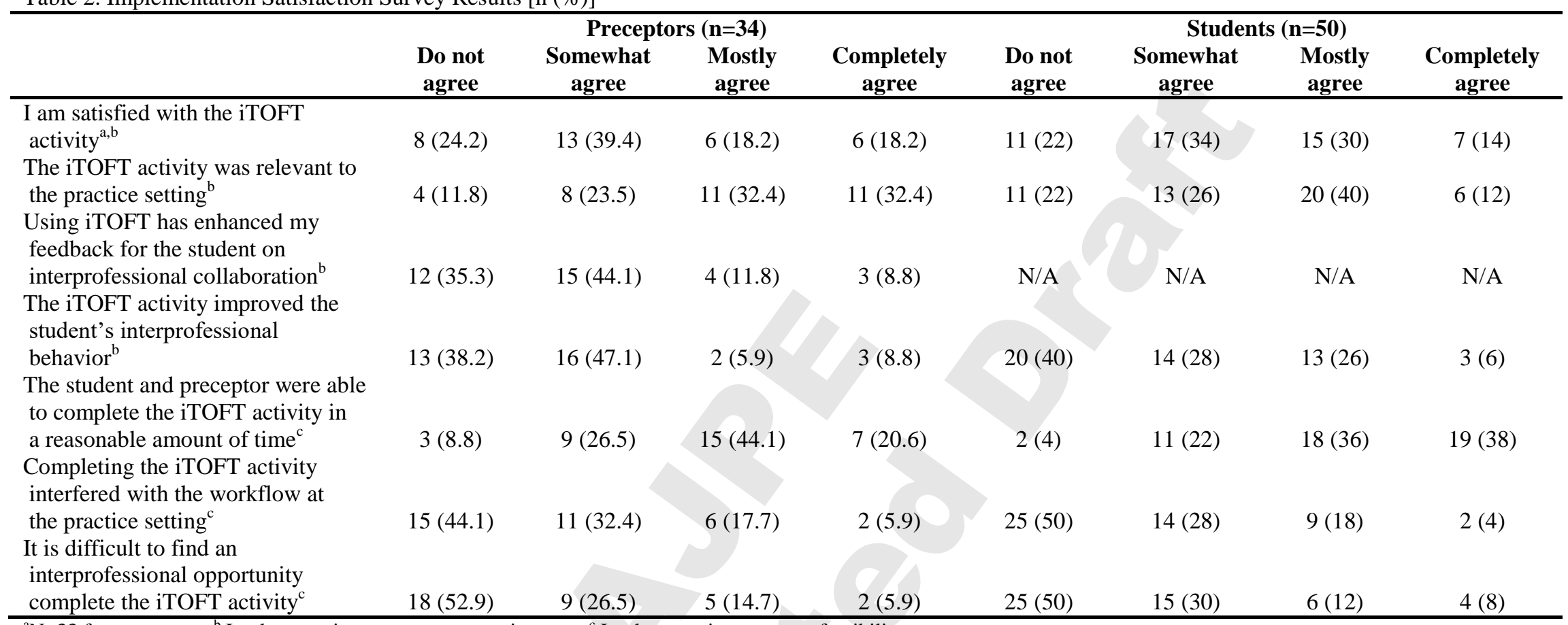

activity

${ }^{\mathrm{a}} \mathrm{N}=33$ for preceptors; ${ }^{\mathrm{b}}$ Implementation outcome appropriateness; ${ }^{\mathrm{c}}$ Implementation outcome feasibility

iTOFT=Individual Teamwork Observation and Feedback Tool 


\begin{tabular}{lcc}
\hline \multicolumn{1}{c}{ Activity } & $\begin{array}{c}\text { Preceptor Reported } \\
\text { Completed (n=33) }\end{array}$ & $\begin{array}{c}\text { Student Reported } \\
\text { Completed (n=50) }\end{array}$ \\
\hline Watch iTOFT training & $25(75.8)$ & $24(48)$ \\
Schedule specific direct observation for activity & $6(18.2)$ & $6(12)$ \\
Preceptor observed performance directly & $27(81.8)$ & $31(62)$ \\
Preceptor conducted more than 1 observation & $13(39.4)$ & $11(22)$ \\
Student completed a self-evaluation of the iTOFT & N/A & $13(26)$ \\
Student and preceptor discussed iTOFT self-evaluation & $22(66.7)$ & $16(32)$ \\
Student and preceptor discussed interprofessional practice & N/A & $33(66)$ \\
Preceptor gave feedback from the iTOFT & $22(66.7)$ & $30(60)$ \\
\hline iTOFT=Individual Teamwork Observation and Feedback Tool & &
\end{tabular}

